\title{
Combined diabetic treatment with low carbohydrate diet, exercise and music therapy
}

\section{Hiroshi Bando* \\ Tokushima University and Medical Research, Tokushima, Japan}

Recently, patients with metabolic syndrome such as obesity, diabetes, hypertension and hyperlipidemia have been increasing worldwide and nutritional therapy is indispensable. There has been discussion and controversy about Calorie Restriction (CR) and Low Carbohydrate Diet (LCD) for years. Originally, LCD was started by Atkins and Bernstein [1-3], and author and colleagues have started and developed LCD in Japan by books, seminars and workshops through Japan Low Carbohydrate Diet Promotion Association. Three kinds of LCD meal were simple and useful for understanding and developing of LCD, which are super, standard and petit LCD [4]. We have also continued clinical researches concerning ketone bodies, Morbus (M) value, comparison of CR and LCD and insulinogenic index (IGI) for CR breakfast including $70 \mathrm{~g}$ of carbohydrate $[5,6]$.

Among our reports, representative data of CR and LCD would be as follows [7]: patients with type 2 diabetes mellitus (T2DM) were divided into 3 groups (low, middle, high) by HbAlc value with $6.1 \%$, $7.9 \%, 9.3 \%$, respectively. Meals were provided CR on day 1,2 , and LCD on day 3-12. Comparison of the value on day 2-day 12 in each 3 group revealed that fasting glucose was $109-98,155-121,211-130 \mathrm{mg} / \mathrm{dL}, \mathrm{M}$ value was $26-10,94-19,343-84$, and Triglyceride was 106-70, 121-83, $164-125 \mathrm{mg} / \mathrm{dL}$, respectively. $M$ value means the average glucose and mean amplitude of glycemic excursions (MAGE) in a day [8]. These results suggested the clinical efficacy of LCD for glucose variability and lipid metabolism in short period with only in 10 days.

Treatment for diabetes needs to include and continue nutritional therapy such as CR and LCD, and also exercise therapy [9]. The latter includes two factors. One is fundamental physical activity and movement in our daily lives with usual Activities of Daily Living (ADL) and Quality of Life (QOL), and another is aerobic, anaerobic or mixed physical exercise and additional sports. Usually diabetic patients tend to be not so active physically, then we have to advise them to move around and/or challenge pleasant and light degree of physical activity and any sports.

Furthermore, we have used some effective therapy for diabetic patients from several treatments and care for Integrative Medicine (IM) and Complementary and Alternative Medicine (CAM), such as physiotherapy and music therapy. The author is not only the physician, but also pianist, music therapist and sports doctor. Then, we have given patients for integrated combination of treatment with the cooperation of our related staffs. We have 3 useful options.

Firstly, stretching procedure with pole exercise would become the key connecting from preparation movement to exercise $[10,11]$ (Figure 1a). Just after performing pole exercise as an introductory physical movement, any favorite activity or sports would be expected consecutively.
Secondly, effective way to continue physical movement with fun is recommended. It is walking, jogging or running outside with listening to favorite music by wearing 'walkman'. There has been the coupling theory in the field of physiotherapy and sports medicine. When 3 factors (rhythm of gait frequency, heart beat and respiration rate) would have successfully synchronized condition, the subjects can run or jog on rapid speed and/or on long slow distance (LSD) comfortably (Figure 1b).

Thirdly, another application of music therapy would be some activities of exercise inside the room associated with music. Wellknown activity is aerobic exercise and pedaling movement using bicycle. In the latter, accompany of music or intermittent beep sound would be helpful for easier repetition of exercise movement. This principle is called as neuromuscular synchronization [12]. The reason is that pedaling training system is using auditory feedback, and that the effect of rhythmic auditory stimulus (RAS) on gait cycle will be also used for the treatment of Parkinson's disease [13].

Application of music and exercise therapy for diabetic patients would be clinically simple, and be expected for continuation of

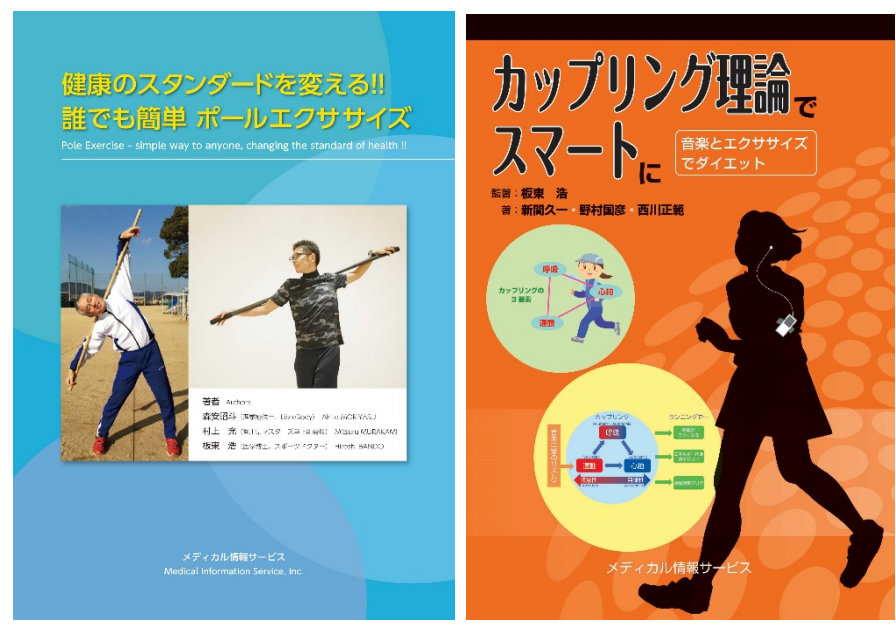

Figure 1. Books of education for diabetic patients (a) Pole Exercise - simple way to anyone, changing the standard of health !! (2018) (b) Be smarter physically and mentally by coupling theory (2010)

${ }^{\star}$ Correspondence to: Hiroshi Bando, $\mathrm{MD}, \mathrm{PhD}, \mathrm{FACP}$, Tokushima University and Medical Research, Nakashowa 1-61, Tokushima 770-0943 Japan, Tel: +8190-3187-2485; E-mail: pianomed@bronze.ocn.ne.jp

Received: July 24, 2018; Accepted: July 30, 2018; Published: August 02, 2018 
treatment and care with ease. Among lots of treatment of IM and CAM, music therapy has been mostly accepted and evaluated for everyone [14].

Moreover, another recommendation of CAM treatment for diabetic patient is spa therapy, marine therapy or hot and cold thermal therapy. After some exercise, bathing with refreshing would be proposed. If the patient feels comfortable in spa therapy after physical exercise, we can expect that he will make a habit of exercising every day or regular time for sports per week.

Regarding recent tendency of treatment of diabetes, the beneficial effect of LCD has been reported $[15,16]$ and some changes in the guidelines were found $[17,18]$. For the decision of therapy for diabetic patients, integrated judgement from broad options of treatment and care would be taken into consideration for optimal ADL and QOL.

In summary, diabetic treatment for patients with diabetes was discussed in this report. There are several options for nutritional therapy, exercise therapy, and others such as music therapy, physiotherapy, spa therapy and so on. This article would become one of the reference to describe the possibility of combined various strategy for diabetic treatment.

\section{References}

1. Atkins and Robert (1996) Dr. Atkins' New Carbohydrate Gram Counter. M. Evans and Company.

2. Bernstein RK (1997) Dr. Bernstein's Diabetes Solution. Little, Brown and company, New York.

3. Ebe K, Ebe Y, Yokota S, Matsumoto T, Hashimoto M, et al. (2004) Low carbohydrate diet (LCD) treated for three cases as diabetic diet therapy. Kyoto Medical Association Journal 51: 125-129.

4. Bando H, Ebe K, Muneta T, Bando M, Yonei Y (2017) Clinical effect of low carbohydrate diet (LCD): case report. Diabetes Case Rep 2: 124.

5. Muneta T, Kawaguchi E, Nagai Y, Matsumoto M, Ebe K, et al. (2016) Ketone body elevation in placenta, umbilical cord, new born and mother in normal delivery. Glycative Stress Res 3: 133-140.
6. Bando H, Ebe K, Muneta T, Bando M, Yonei Y (2017) Proposal for insulinogenic Index (IGI)-Carbo70 as experimental evaluation for diabetes . J Clin Exp Endocrinol 1: 102 .

7. Bando H, Ebe K, Muneta T, Bando M, Yonei Y (2017) Effect of low carbohydrate diet on type 2 diabetic patients and usefulness of M-value. Diabetes Res Open J 3: 9-16.

8. Service FJ, Molnar GD, Rosevear JW, Ackerman E, Gatewood LC, et al. (1970) Mean amplitude of glycemic excursions, a measure of diabetic instability. Diabetes 19: 644655. [Crossref]

9. Ebe K, Bando H, Yamamoto K, Bando M, Yonei Y (2018) Daily carbohydrate intake correlates with HbAlc in low carbohydrate diet (LCD). J Diabetol 1: 4-9.

10. Moriyasu A, Bando H, Murakami M, Inoue T, Taichi A, et al. (2018) Pole exercise causes body changes in physical flexibility and exercise function. $J$ Nov Physiother 8: 377 .

11. Moriyasu A, Bando H, Akayama R, Wakimoto K, Dakeshita T, et al. (2017) Thorax flexibility can be increased by standing pole exercise. Int J Phys Med Rehabil 6:1.

12. Zhang J, Zhang K, Feng J, Small M (2010) Rhythmic dynamics and synchronization via dimensionality reduction: application to human gait. PLoS Comput Biol 6: e1001033. [Crossref]

13. Chapman AR, Vicenzino B, Blanch P, Knox JJ, Dowlan S, et al. (2008) The influence of body position on leg kinematics and muscle recruitment during cycling. J Sci Med Sport 11: 519-526. [Crossref]

14. Bando H, Yoshioka A, Nishikiori Y (2018) Music Therapy Session in the Hospital would Relax, Sooth and Heal the Heart. J Integrative Med Ther 5: 1.

15. Shai I, Schwarzfuchs D, Henkin Y, Shahar DR, Witkow S, et al. (2008) Weight loss with a low-carbohydrate, Mediterranean, or low-fat diet. N Engl J Med 359: 229-241. [Crossref]

16. Dehghan M, Mente A, Zhang X, Swaminathan S, Li W, et al. (2017) Prospective Urban Rural Epidemiology (PURE) study investigators (2017) Associations of fats and carbohydrate intake with cardiovascular disease and mortality in 18 countries from five continents (PURE): a prospective cohort study. Lancet 6736: 32252-3.

17. American College of Physicians (2017) Clinical Guidelines and Recommendations. Web site. http://www.acponline.org/clinical-information/guidelines.

18. American Diabetes Association (2018) Pharmacologic Approaches to Glycemic Treatment: Standards of Medical Care in Diabetes-2018. Diabetes Care. 41:S73-S85. [Crossref]

Copyright: (C2018 Bando H. This is an open-access article distributed under the terms of the Creative Commons Attribution License, which permits unrestricted use, distribution, and reproduction in any medium, provided the original author and source are credited. 